\title{
Tailored approach to sleep health education (TASHE): study protocol for a web-based randomized controlled trial
}

\author{
Natasha J. Williams ${ }^{1 *}$, Rebecca Robbins ${ }^{1}$, David Rapoport ${ }^{2}$, John P. Allegrante ${ }^{3}$, Alwyn Cohall ${ }^{4}$, \\ Gbenga Ogedgebe ${ }^{1}$ and Girardin Jean-Louis ${ }^{1}$
}

\begin{abstract}
Background: Obstructive sleep apnea (OSA) is a sleep disorder that disproportionately affects African Americans (hereafter referred to as blacks). Moreover, blacks may underutilize sleep services including overnight polysomnography. Thus, OSA among blacks may go undiagnosed and untreated, which has significant health consequences, including hypertension, diabetes, cognitive impairment, and daytime sleepiness.
\end{abstract}

Design and Methods: This two-arm randomized controlled trial will assign 200 participants to a culturally and linguistically tailored web-based sleep educational platform. The website will be developed to ensure that the content is user friendly and that it is readable and acceptable by the target community. Participants will receive login information to a password-protected website and will have access to the website for 2 months. Study assessments will be collected at baseline, 2 months (post-enrollment) and at 6 months (follow-up). We will use qualitative and quantitative methods to develop tailored materials and to ascertain whether tailored materials will increase OSA knowledge and OSA health literacy by comparing blacks exposed to tailored materials versus those exposed to standard sleep health literature. We hypothesize that exposure to tailored OSA information will improve OSA health literacy.

Discussion: Few studies have investigated the racial/ethnic disparities in relation to OSA screening and treatment comparing blacks and whites. Moreover, we know of no interventions designed to increase OSA knowledge and health literacy among blacks. Use of the Internet to disseminate health information is growing in this population. Thus, the Internet may be an effective means to increase OSA health literacy, thereby potentially increasing utilization of sleep-related services in this population.

Trial registration: The study is registered at clinicaltrials.gov, reference number NCT02507089. Registered on 21 July 2015.

Keywords: Adherence, Sleep health, Blacks, Continuous positive airway pressure, Health education, Health literacy, Internet, Obstructive sleep apnea

\section{Background}

Increasingly, researchers and practitioners recognize that obstructive sleep apnea (OSA) - one of the most commonly diagnosed sleep disorders - disproportionately affects blacks. One of the earliest studies documenting racial/ethnic differences in the rate of OSA compared 225

\footnotetext{
* Correspondence: Natasha.williams2@nyumc.org

'Department of Population Health, Division of Health and Behavior, Center for Healthful Behavior Change, NYU Medical Center, New York, NY 10016, USA

Full list of author information is available at the end of the article
}

black and 622 white volunteers, ages $2-86$ years, and found that $31 \%$ of blacks versus $10 \%$ of whites had OSA [1]. More recent studies have also reported similar disparities including adherence to OSA treatment, with blacks using positive airway pressure (PAP) far less than their white counterparts [2-4]. The underlying mechanisms of these disparities are not clear. However, research suggests that the disparities in OSA prevalence may be explained by genetic factors and obesity [5], while disparities in treatment adherence may be partially explained by socioeconomic status [3] and sleep duration [2]. 
The Institute of Medicine and the National Institutes of Health (NIH) recognize OSA as a chronic disease that requires novel adherence strategies to promote enhanced quality of life and diminish social and economic costs [6]. Yet, our pilot data suggests that blacks tend to underutilize sleep services. This is unfortunate given that there may be a high prevalence of OSA in this community, and that treatment is effective in improving cardiovascular disease (CVD) risk factors, especially blood pressure [7], which disproportionately burden blacks.

\section{Insufficient sleep and sleep disorders}

A recent analysis of data from 12 states conducted by the Centers for Disease Control and Prevention (CDC) illustrated that $35.3 \%$ of United States (US) adults reported insufficient sleep and $37.9 \%$ reported unintentionally falling asleep during the day [8]. It also appears that there is increased awareness about sleep in the general population. For example, using the National Ambulatory Medical Care Survey, Ford et al. [9] found a 13\% increase in the number of physician office visits for sleep complaints and a $200 \%$ increase in the number of individuals who reported a sleep disorder diagnosis including OSA, insomnia, and restless leg syndrome. As there appears to be an increase in sleep complaints and diagnoses of sleep disorders, as well as the rapidly growing field of sleep medicine, it is paramount that patients be educated about sleep health, including the importance of screening and adhering to treatment, especially among minority populations who may underutilize sleep services. Blacks as well as other racial/ethnic groups with untreated OSA may be at greater risk for hypertension [10], diabetes [7], cognitive impairment [11], and poor quality of life [12].

\section{Sleep and health literacy}

Health literacy is defined as "the degree to which individuals can obtain, process, and understand basic health information, and services they need to make appropriate health decisions" [13]. Health literacy requires several skills including print literacy (reading and writing), numeracy (recognizing numbers in the case of a prescription), and oral literacy (listening and speaking). It is estimated that 77 million Americans have limited health literacy, costing the healthcare system $\$ 106-238$ billion a year [14].

Limited health literacy is a major problem in the US, and health literacy is linked to delayed diagnoses, lack of understanding of medical condition, lack of treatment, poor self-management skills, and worse clinical outcomes [15]. Greater rates of limited health literacy are generally reported among minorities, the elderly, and individuals with low educational attainment. A systematic review indicated that patients with low health literacy were generally 1.5 to 3 times more likely to experience poor health outcomes [16].
Patients with limited health literacy may experience difficulty locating providers and services, completing complex health forms, sharing their medical history with providers; they may also be unaware of the link between health risk behavior and poor health outcomes. It is plausible, then, that describing symptoms of sleep disturbance, undergoing screening for sleep disorders, and adhering to treatment, particularly PAP, would require high-level literacy skills. In a cohort of men from Australia who underwent polysomnography, low functional health literacy, another measure of health literacy, was one of the main factors associated with previously undiagnosed OSA $(\mathrm{OR}=2.43,95 \% \mathrm{CI}=1.40-4.20)$ compared to men with adequate functional health literacy [17]. These findings also suggest that health literacy is associated with the uptake of OSA screening.

The Centers for Medicare and Medicaid Services requires that the patient use PAP for 4 or more hours per night, $70 \%$ of nights in a 30 -day period for reimbursement [18]. In addition to the lack of empirical evidence to support this guideline as a necessary and sufficient marker of adherence, this information might be difficult for patients to comprehend; patients may be unable to process the amount of time that they are required to use their PAP mask accurately in order to achieve adherence. In addition, adherence to PAP may require a high level of oral communication skills whereby patients should be able to communicate with their provider whether or not they are experiencing challenges with mask use; the PAP mask may involve several challenges including negative side effects and mask discomfort [19]. It is plausible that some of the adherence issues may be exacerbated when coupled with mental health problems, and there is a high rate of depression among patients diagnosed with OSA. In effect, in an analysis of the 20052008 National Health and Nutrition and Examination Survey, Wheaton et al. [20] reported that the rate of depression was two and five times more likely to be reported among men and women, respectively, who were diagnosed with OSA. Thus, adhering to physician recommendations for OSA screening and treatment requires a high degree of health literacy, and there may be significant consequences for those patients with low literacy skills.

Similarly, patients may have difficulty completing commonly used sleep surveys. One study found that $56 \%$ of new sleep patients had difficulty completing the Epworth Sleepiness Scale, a commonly used measure for describing sleep symptoms [21]. In another study, $44 \%$ of patients with low literacy skills required assistance reading $\mathrm{Na}$ tional Sleep Foundation (NSF) and American Academy of Sleep Medicine (AASM) brochures [22]. Using the Rapid Estimate of Adult Literacy in Medicine [23] questionnaire, a survey of patients from 122 sleep centers showed that 
$16.3 \%$ exhibited impaired health literacy [21]. Of note, the Center for Health Care Strategies does not recommend such tools in communities characterized by low literacy levels and trust [24-27], unless these materials are specifically tailored for the intended community [28].

\section{Objectives}

Despite these limited studies, which have significant implications for the field of sleep medicine, we know of no interventions to improve adherence to treatment for sleep disorders or adherence to physician recommendations for sleep screening among patients with low health literacy. Our primary objective is to develop the Tailored Approach to Sleep Health Education (TASHE) program using a multilevel community-engaged approach. We will use themes from focus group to develop a culturally and linguistically tailored wbsite and video vignettes. We will incorporate those themes into an interactive website with OSA health content designed for lay people. This website will thus serve as a platform facilitating exchange of information between sleep scientists, community health providers, potential patients, and lay health advisors. We will also seek endorsement of TASHE materials by healthcare providers and stakeholders to ensure their adoption and dissemination. Our secondary objective is to evaluate TASHE's effectiveness in increasing OSA health literacy among blacks. The main hypotheses for this study are: (1) modifiable barriers (e.g., negative beliefs and attitudes toward sleep, perceived bias in healthcare, inadequate access, and lack of cultural and linguistic tailoring of sleep health materials to blacks) will comprise emergent themes from focus groups; (2) participant engagement will increase acceptability of sleep health messages. Stakeholder engagement will facilitate adoption, dissemination, and sustainability of the program. Provider engagement will enable tailoring of messages addressing patient's concerns about effectiveness, benefits, and harms of treatment options to inform decision-making; and (3) exposure to tailored OSA health messages will improve OSA health literacy.

\section{Design and methods}

This two-arm randomized controlled trial will include use of an interactive, culturally and linguistically tailored website for blacks at risk of OSA. The secondary objective is to increase knowledge about OSA and improve OSA health literacy. The approach was designed based on the formative evaluation that was conducted in the NIHfunded study 'Metabolic Syndrome Outcome Cohort' study $[29,30]$ in which results from focus groups included misperceptions about OSA and mistrust of the healthcare system [31, 32]. Results of that study suggested the need to use an innovative approach to increase OSA knowledge and OSA health literacy via a web-based platform.

\section{Study design and overview}

\section{A community-engaged approach}

We will bring together academic investigators, community providers, and stakeholders, which is considered to be an effective tool to develop interventions, sustainable programs, and policies to address health disparities, and to improve adoption of healthful practices in underserved communities [33, 34]. This partnership approach begins with a research topic of importance to the community (i.e., sleep apnea) and combines knowledge with action to achieve social change in order to improve health outcomes and eliminate health disparities [35]. It is becoming increasingly evident that community engagement in the research process is critical. Doing so leads to identification or refinement of relevant research questions [36], delineates barriers, increases community participation in research [37], improves effectiveness of interventions [38], and enhances dissemination of study results [36] (see Fig. 1).

\section{Faculty advisory board}

We will convene a Faculty Advisory Board (FAB) comprised of the leading sleep medicine physicians, scientists, and researchers, which will be tasked to help develop (review and approval) the sleep health content of all TASHE materials before dissemination. This will ensure that materials emphasize current concepts and understanding regarding sleep health and circadian and sleep biology and promulgate important findings of the National Heart, Lung, and Blood Institute (NHLBI)funded clinical trials.

\section{Community steering committee}

Our experience conducting minority research has informed us that interventions are best poised to be effective and sustainable when they are culturally appropriate and responsive, and that stakeholders in the partner community endorse them, such that the end result is increased empowerment of community members to address their own health issues. Community academic partnerships, as we have assembled to achieve goals of the TASHE project, have significant advantages over efforts undertaken by researchers alone because such partnerships bring a diversity of expertise that facilitates a more comprehensive understanding of community strengths and needs, as well as improved capacity to plan, execute and evaluate the project. We will create a Community Steering Committee (CSC) [39] bringing together community stakeholders, patients, and health advocates to work with the TASHE project leadership and the FAB. In addition, the CSC will provide feedback on the content of the website to ensure that it reflects the voice of the community and to provide feedback on other programmatic areas including design features and 


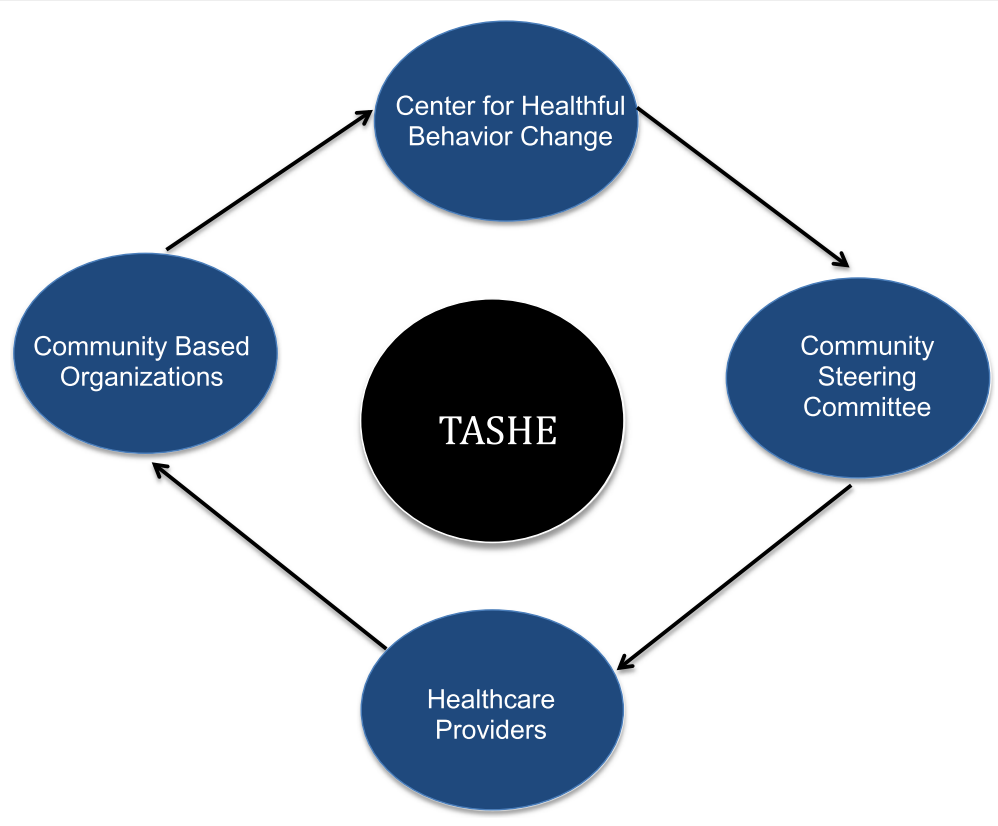

Fig. 1 Multi-level Community-engaged approach

content of the website. The CSC will receive a summary of all research-related findings including themes from the focus groups and preliminary study results throughout the study period.

\section{Website development}

The content of the website will be based on several resources: (1) the FAB and members of the investigative team will advise on the content that should be included and develop all necessary informational sections; (2) a literature review; and (3) a review of existing sleeprelated websites and brochures including the AASM, the NHLBI, and the NSF. The FAB will review the content for accuracy and completeness. The content will then be presented to the CSC, which will provide additional suggestions and comments; these will be incorporated into the final content used in the website. The investigative team will review the content to assess health literacy including ease of use, design layout, and readability. We will use the Flesch-Kincaid readability formula and the material will be customized at the fifth-sixth grade reading level.

\section{Tracking and Internet metrics}

The website will be password-protected, accessible only by participants in the exposure arm. By providing a specific password to each participant, we will be able to track their activity on the site using Google Analytics logfile data and relate it to other participant characteristics. Google Analytics is a web analytics service offered by Google that tracks website usage and traffic. We will use this resource to ascertain how participants use the website by linking their login details with metrics generated by Google including pages visited, duration of time on each page, and frequency of visits to the website.

\section{Role model storytelling development}

The primary vehicle by which the project will achieve its goal is through role model storytelling video vignettes. Evidence suggests that video presentation of OSA materials to patients with low literacy skills helped improve OSA knowledge (66\% vs. $43 \%)$ and usage of continuous PAP (94\% vs. $78 \%$ ) [40]. Video vignettes acquired from interviews with black patients with OSA will demonstrate how poor sleep affects one's daily functioning and overall health and quality of life; barriers and facilitators to adhering to OSA treatment; undergoing an overnight in-lab and in-home sleep study; and discussing treatment options with a sleep provider. We will use Taylor's model as the framework guiding the role model story development. According to Taylor's model [28], role model stories are effective if they reflect realities of individuals from the community to which they are directed. To be credible, the role model will reflect the race, culture, and socioeconomic circumstances of community members. Thus, the appropriate role model will be someone from that community who has adopted recommended healthful practices to be modeled in the story. Thus, initially potential role models recruited from the community will be screened in order to determine if the person could provide enough information about his/ her experiences to create a role model story. 


\section{Heurestic testing and tailoring}

All role model stories and video vignettes included in the TASHE website will be pretested before randomization. We will conduct heuristic evaluation, led by a member of the investigative team, and cognitive walkthroughs of all web materials, and then perform standard formative usability testing with eight to ten representative participants to ensure the site is well-suited to the needs of the intended community. We will draw upon the work of Kreuter and colleagues [41] that outlines several steps on the way toward the design of tailored materials for health promotion activities. Developing tailored materials is an iterative process whereby participant and population needs are identified, formative materials are designed, feedback from the community or population is obtained, then another round of edits are made before materials can truly be termed 'tailored'. After the opinions, attitudes, and beliefs about sleep and OSA specific to the population are ascertained, the website will be available for the intervention.

Data obtained from the usability testing include qualitative data obtained from the interviews, as well as quantitative metrics obtained from the computer-tracking software. The data analyst will conduct a qualitative analysis to identify themes in the participant reactions and comments. In addition, the quantitative data collected vis-à-vis the software program will be analyzed using statistical software. Descriptive analysis for the various quantitative metrics will be tabulated to understand the various website components that drew the most attention from participants.

\section{Focus groups}

For aims 1 and 2 of this study, the focus will be on determining barriers preventing uptake of healthful sleep practices in the partner community and developing health messages to promote uptake of these practices. Barriers will be elicited through focus groups, and tailored health messages will be developed and refined. We will conduct five focus groups with patients and community stakeholders. In the first three groups, we will document barriers and factors facilitating or inhibiting uptake of healthful sleep practices. Emergent themes will be presented to the next two groups; here, the primary focus will be on reactions to these themes. Targeted themes will include beliefs, knowledge, values about healthful sleep practices, and mediating or reinforcing factors associated with healthful behavior. We will also conduct focus groups with community stakeholders and with healthcare providers. The purpose of the stakeholder focus group will be on reactions to health messages developed based on themes emanating from focus group with participants in aim 1. During the provider interviews, we will gauge their understanding of participants' perspectives on benefits and harms of preventive, diagnostic, therapeutic, or sleep healthcare delivery systems. These factors influence participants' decisionmaking regarding uptake of healthful practices.

\section{Eligibility criteria}

Participants must meet the following study criteria: inclusion criteria: self-reported race/ethnicity as African American, African, Caribbean American or black men and women; ages $\geq 18$ years; accessible by telephone; no plans to move away from the region within the year following enrollment; consent to participate, which includes permission to release medical record information; documented OSA risk based on scores received from the Apnea Risk Evaluation System (ARES) questionnaire [42]. Internet access is not an eligibility criterion, as participants will receive an iPad with cellular service.

Exclusion criteria will include: progressive medical illness in which disability or death is expected within 1 year; impaired cognitive or functional ability which would preclude meaningful participation in the study; sleep apnea diagnosis; stated intention to move within the same year of enrollment.

\section{Recruitment}

We will use several recruitment strategies. First, we will utilize a recruitment funnel that we have developed from several other Institutional Review Board (IRB)-approved $\mathrm{NIH}$-funded studies related to sleep health. Participants will be contacted via phone to ascertain their level of interest. If participants express interest, they will be invited to come to our office at New York University. Then, a member of the research team will provide detailed information about the study protocol, and read the consent form. Participants will have time to ask questions.

Second, we will host information sessions with prospective participants. These sessions will last approximately 20 minutes and will be led by a senior member of the investigative team. The purpose of the session is to (1) provide detailed information about the study proto$\mathrm{col}$, (2) discuss requirements and expectations of participants, and (3) ascertain interest in moving forward with the study. Participants will receive information materials and a consent form that they may take home to discuss with their family, friends, or doctor, before they agree to sign the consent form. This will allow participants enough time to make an informed decision about whether or not to participate in the study. Participants will have time to answer questions of the investigative team.

Third, we will rely on established relationships with community-based and faith-based organizations including barbershops and churches. Through these efforts, the research team is able to go on site to gauge participant interests. We will follow the same procedures throughout the study, which involve: (1) providing detailed information about the study, (2) providing study materials for 
participants to review on their own time, and (3) allowing participants enough time to ask questions freely.

Finally, recruitment materials including flyers, brochures, and fact sheets will be developed in conjunction with the CSC. These materials will be at the fifth-sixth grade reading level. All materials provide an overview of what is involved in participating in the study, and will be placed in community venues including barbershops, community-based organizations, and churches. The CSC members will provide feedback to ensure that the materials are clearly written, and that they are acceptable to the community. The Project Manager in consultation with the Principal Investigator (PI) will oversee recruitment and consent.

\section{Allocation}

Individuals will be eligible for randomization once their baseline questionnaire is completed. Randomization will be carried out in pairs using a table of random permutations conducted by the Program Manager. One member of each pair will be assigned to the Internet-based group and the other to the control group, using sealed envelopes. This strategy ensures equal allocation to the two groups, and that both have similar characteristics regarding measured and unmeasured background variables (i.e., patient characteristics and lifestyle behavior at baseline, medical comorbidities, and situational/contextual factors), which could potentially affect study outcomes. The Program Manager will hold the sequence of assignments and will dispense the code for each new patient upon request by the Research Assistant (RA). If a participant requests to be withdrawn from the study, the Program Manager will assign the code for the next new patient. The Program Manager will maintain a record of who was randomized to which group to maintain integrity of planned analysis. Due to the nature of the study, the Program Manager will not be blind to the study arms. Randomization will be ongoing until sample size goals are reached: 100 per study arm and in accordance with the CONSORT randomization guidelines as shown in Fig. 2.

\section{Measures}

Screening of baseline measures: we estimate that completion of the baseline study measures will take up to 15 minutes. For participants who are eligible and enrolled into the study, we estimate that the additional screening questionnaires will take up to 30 minutes.

\section{Sociodemographic variables}

Sociodemographic variables will include age, type of health insurance, marital status, education, number of children living in household, number of adults living in household, employment status, and annual household income.

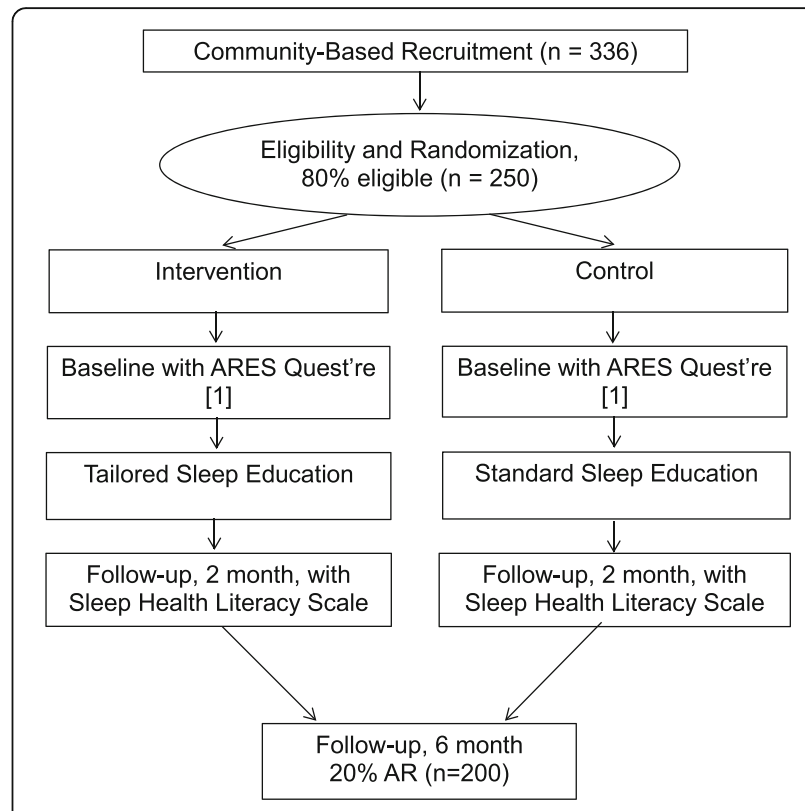

Fig. 2 CONSORT flow diagram

\section{Apnea Risk Evaluation System questionnaire}

The ARES includes questions on sleep patterns, daytime functioning, knowledge of sleep apnea, and diseases associated with sleep apnea [that is, hypertension (HTN), diabetes mellitus (DM), heart disease] [42].

\section{Epworth Sleepiness Scale}

The Epworth uses a Likert-type scale in which the respondent indicates the most appropriate number ranging from $0=$ would never doze or sleep to $3=$ high chance of dozing or sleeping based on a given situation. A score of 10 or more is considered excessive sleepiness [43].

\section{Apnea Knowledge Test (AKT) [44] and Apnea Beliefs Scale (ABS) [44]}

These two questionnaires measure participants' understanding and beliefs of obstructive sleep apnea and CPAP treatment. The AKT includes 13 questions with a yes/no response choice and two open-ended questions; the ABS includes 24 items on a five-point Likert scale ranging from "strongly agree" to "strongly disagree". A higher score on the AKT indicates better knowledge and understanding of the illness and treatment and a higher score on the ABS indicates greater likelihood of adherence to treatment [44].

\section{Change Assessment Scale (CAS)}

The CAS is a 32-item instrument that assesses a person's readiness to change. Participants evaluate the extent to which they strongly agree or strongly disagree with various statements such as, "I've been thinking that I might 
want to change something about myself" and "I have problems and I really think I should work on them."

\section{Medical history}

Medical history includes family history of sleep disorders, obesity, DM, HTN, dyslipidemia, and CVD; history of other preventive behaviors (for example, prostate examinations), and other health behaviors (for example, regularity of physical examinations, cholesterol screening, smoking and drinking habits, and exercise habits) [45].

\section{Sleep Hygiene Index (SHI)}

The SHI is a 13-item instrument that measures sleep hygiene behaviors. It was derived from the diagnostic criteria for inadequate sleep based on the International Classification of Sleep Disorders. Participants are asked about the frequency that they engage in specific behaviors with a response of "always," "frequently," "sometimes," "rarely," or "never." Behaviors such as using the bed for things other than sleeping and sex, and daytime naps longer than 2 hours are included [46].

\section{Short Test of Functional Health Literacy in Adults (S- TOFHLA)}

The S-TOFHLA is a measure of a patients reading and comprehension of both print and numeracy health-related information [23].

\section{Sleep Apnea Literacy Scale}

This is a 24-item instrument designed to measure patient-level knowledge, efficacy, and literacy barriers to effective treatment for sleep apnea. Specific items include statements such as "when breathing stops during sleep this is called sleep apnea" and "poor concentration can be caused by sleep apnea." Participants are asked to mark their confidence for these statements about sleep apnea on a scale from "very confident this is true" to "not at all confident this is true" [47].

\section{Internet Self-Efficacy Scale}

This scale will measure confidence in the ability to use the Internet. Participants rate their confidence on a Likerttype scale and are asked statements such as, "using the Internet to gather data" and "turning to an online discussion group when help is needed" [48].

A complete list of measures and the data collection schedule is provided in Table 1.

\section{Intervention}

Participants randomized to the intervention arm will receive an iPad with free access to a password-protected interactive culturally and linguistically tailored sleep informational website. Study assessments will be collected at baseline, 2 months (post-enrollment) and at 6 months
Table 1 Study measures and schedule to acquire data

\begin{tabular}{|c|c|c|c|}
\hline \multirow[b]{2}{*}{ Measures } & \multicolumn{3}{|c|}{ Time point } \\
\hline & Baseline & 2 months & 6 months \\
\hline Demographic and clinical variables & $x$ & & \\
\hline Medical Outcomes Study Short Form 36 & $x$ & & \\
\hline Self-Efficacy Scale & $x$ & $x$ & $x$ \\
\hline Change Assessment Scale & $x$ & $x$ & $x$ \\
\hline Intrinsic Motivation & $x$ & $x$ & $x$ \\
\hline $\begin{array}{l}\text { Apnea Risk Evaluation System } \\
\text { Questionnaire }\end{array}$ & $x$ & & \\
\hline Sleep Hygiene Index & $x$ & $x$ & $x$ \\
\hline Apnea Knowledge/Apnea Belief Scale & $x$ & $x$ & $x$ \\
\hline Sleep Apnea Literacy Scale & $x$ & $x$ & $x$ \\
\hline $\begin{array}{l}\text { Test of Functional Health Literacy } \\
\text { in Adults (short version) }\end{array}$ & $x$ & $x$ & $x$ \\
\hline Internet Self-Efficacy Scale & $x$ & $x$ & $x$ \\
\hline
\end{tabular}

(follow-up). Participants will receive a brief 10-minute tutorial by the study team on login procedures and use of the website. Participants will also be able to call the project staff to troubleshoot any issues with the website. Given that this is a web-based intervention, the study setting is the participants' home.

\section{Control condition}

Participants randomized to the control condition will receive an iPad with free access to the NSF and NHLBI website via an iPad. Currently, there is no specific comparative effectiveness data evidencing superiority of any website or other format for increasing sleep health literacy. Furthermore, there has been previous research to suggest the overall difficulty that patients experience in using the NSF materials. Thus, our study will ascertain effectiveness of a tailored website compared to the standard websites. Participants in the control condition will have access to the culturally and linguistically tailored sleep informational website at 6 months postenrollment date.

\section{Design and methods: data collection, management, and analysis}

Survey data will be collected through a passwordprotected website, REDCap, a secure website for managing and collecting online survey data, which meets the standards set forth by the NYULMC IRB and HIPPA offices. Additional information about data entry, storage, quality and can be found in the IRB study protocol.

\section{Plans to promote retention}

Participants in the intervention arm will be contacted weekly by the PM to ensure ongoing use of the website 
and access to intervention materials. We will track participant usage based on Google Analytics.

\section{Sample size}

Guided by principles governing the use of focus group data, the sample size will be determined when we reach a point of "saturation", which is when additional group sessions are not likely to produce new themes. Based on previous research $[49,50]$, we expect that five groups will be sufficient to capture key themes for the project. In the secondary objective - assuming a medium effect size $(\mathrm{d}=0.32)$, as derived from Cohen [51], and a sample size of 200 - the study will be adequately powered (>85\%) to detect significant differences between participants likely to show improvement in health literacy scores and those who are not using Fisher's exact tests (critical $\mathrm{X}^{2}=7.98$ ). In a previous pilot study, we found that $40 \%$ of black patients in the community adopted recommendations for OSA treatment after exposure to health messages, which compares to $27 \%$ who did without such exposure. Assuming a 13\% increase in the likelihood of adoption of health messages and a sample size of 200, the proposed study will have adequate power for a preliminary test of the hypothesis that exposure to health messages will foster improvement in sleep health literacy. Achieving the goal of a $13 \%$ increase would have an important public health impact. A two-tailed test, with $85 \%$ power and $\alpha=0.05$ comparing $27 \%$ to $40 \%$ requires 100 participants per arm [52].

\section{Statistical analysis}

Zemke and Kramlinger's procedures will be used to analyze qualitative data for aims 1 and 2. Focus groups will be recorded and transcribed verbatim. Transcripts will be reviewed line by line and assigned codes or labels to develop themes. Transcripts will be coded and reviewed by two independently trained RAs and the study team will review transcripts in case of any disagreement with codes and themes. For the qualitative data our first hypothesis is that modifiable barriers (e.g., negative beliefs and attitudes toward sleep, perceived bias in healthcare, inadequate access, and lack of cultural and linguistic tailoring of sleep health messages to minorities) will be consistent themes in focus groups. For hypothesis 2: participant engagement will increase acceptability of health messages depicted in posters and dispensed through the interactive website. Stakeholder engagement will facilitate adoption, dissemination, and sustainability of the program. Provider engagement will enable tailoring of health messages addressing participant's concerns about effectiveness and benefits of different treatment options to inform decision-making. Since we plan to analyze both qualitative and quantitative data, we'll adhere to Office of Behavioral and Social Sciences
Research (OBSSR)-recommended 'Best Practices for Mixed Methods Research in the Health Sciences'.

For aim 3, we will examine which subjective measures are associated with increased OSA health literacy using multivariate logistic regressions. The main dependent measure will be a binary factor: [yes versus no]; candidate predictors are indicated in Table 1 . We will use multivariate analysis to explore whether sociodemographic variables (e.g., age, income, education), lifestyle practices (e.g., diet, exercise, smoking, and drinking habits), Internet self-efficacy, and baseline sleep measures (e.g., OSA knowledge, OSA beliefs, and knowledge of sleep hygiene) indexing OSA health literacy improved after exposure to health messages. We assess OSA health literacy with the Sleep Apnea Literacy scale, a 24 -item questionnaire developed by a sleep expert, and expert in scale design. The questionnaire assesses sleep apnea knowledge and sleep apnea clinical management. Sample items include "When breathing stops during sleep this is called sleep apnea" and "Loud snoring can be a sign of sleep apnea." Ninety-one participants average age 38, and 48\% white, 27\% African American completed the questionnaire. The questionnaire was developed using exploratory and confirmatory analyses. The questionnaire includes items across three scales, including sleep apnea literacy (component alpha 0.74), sleep apnea self-efficacy (component alpha 0.76), and sleep apnea clinical management (component alpha $0.65)$. The concurrent scale validity is 0.85 . To compare participants who exhibited improvement in health literacy and those who did not 2 months post-enrollment, $t$ tests and chi-squared tests will be used. Multivariate analysis of covariance will be used to assess effects of sleep health messages on sleep measures (e.g., OSA knowledge, OSA beliefs, knowledge of sleep hygiene, and S-TOFHLA scores); effects of confounders will be adjusted. Baseline subjective factors will be entered in a linear regression model in order to determine which factors are associated with higher health literacy scores. In post hoc analyses, we will also explore whether combined effects of exposure to tailored web-based content, and video vignettes received through the website had greater impact on improvement in sleep apnea literacy scores.

Both selection and attrition biases might occur during data acquisition for aim 3. Selection bias might result from participant's refusal to participate and attrition bias could result in an unbalanced design. Appropriate statistical techniques (e.g., Heckman two-stage approach GEE probit model, and Monte Carlo [53] will be used to address such biases, or problems that might result from missing data. Adequate statistical corrections will be made in final statistical analyses to increase generalizability of the findings. Sociodemographic data obtained from those who did not participate will be used to determine whether they are 
similar to those in the study [54]. Likewise, baseline characteristics of participants who withdrew from the study will be contrasted with completers to determine whether any attrition bias may have occurred.

The Biostatistician will manage study data under the PI's supervision. He will create computerized data collection forms to ensure integrity and accuracy of data entry. He will help to minimize the problem of missing data by resolving omissions and errors as they arise. Throughout the study, the PI will oversee data entry and verification, ensure adherence to coding, and clean and edit protocols. Participant-identifying information will be kept in a locked file cabinet with only numerical identifiers in computer files.

\section{Data monitoring}

In compliance with NIH requirements, we will establish a data safety and monitoring plan (DSMP). The purpose of the DSMP is to ensure the safety of participants and the validity and integrity of the data. In adherence to $\mathrm{NIH}$ policy regarding data sharing, the DSMP will be tasked to develop its data-sharing plan in consultation with an external representative. Data will be made available from the institutions' website once the main findings of the project have been published. The plan will incorporate language to ensure that the rights and privacy of people who participate in $\mathrm{NIH}$-sponsored research are protected at all times. Thus, data will be free of identifiers that would permit linkages to individual research participants and variables that could lead to deductive disclosure of individual participants. The DSMP internal committee will review all requests for data before access to the data is granted.

\section{Discussion}

OSA is one of the most commonly diagnosed sleep disorders that disproportionately affect blacks. With the rapidly changing field of sleep medicine, including the use of home-based sleep studies, patients may require assistance navigating sleep health services [55]. In addition, there are various treatment options, although CPAP is the most effective. Moreover, treatment for OSA is efficacious and can reduce symptoms of co-morbid health conditions including CVD, diabetes, and hypertension. Unfortunately, adherence to treatment for OSA remains suboptimal and blacks in particular may use CPAP far less than their white counterparts, thus limiting their ability to benefit from treatment. Although several factors have been proposed as contributing to poor adherence, including machine difficulties and psychosocial factors, few studies have explored the role of health literacy $[19,55]$. Limited health literacy is associated with several health outcomes and requires a complex set of skills. Thus, it is plausible that individuals with limited OSA health literacy would benefit from the intervention, which will utilize a web-based approach to increase OSA health literacy that we are testing.

In conclusion, there is growing interest in using telehealth among minorities [56-58]. Studies are beginning to show that Internet use in minority communities has increased over the last several years. A random-digit telephone survey of New York residents found that 77\% had computer access and 65\% used the Internet [59]. Online information affects health habits of New York minorities, with $47 \%$ in a telephone survey stating it changed their exercise routines and $44 \%$, their eating habits [60]. Computer-based pre-screening is well accepted for HIV testing, with $80 \%$ of respondents in one study preferring the computer-based screening compared to other methods [61]. Thus, we believe that an approach that builds on what is now known about the potential role of web-based information might be an effective means to increase OSA knowledge and OSA health literacy, thereby increasing utilization of sleeprelated services among blacks.

\section{Trial status}

Recruiting.

\section{Abbreviations}

AASM: American Academy of Sleep Medicine; ABS: Apnea Beliefs Scale; AKT: Apnea Knowledge Test; ARES: Apnea Risk Evaluation System; PAP: Positive airway pressure; CSC: Community Steering Committee; CVD: cardiovascular disease; DM: diabetes mellitus; DSMP: data safety and monitoring plan; FAB: Faculty Advisory Board; HTN: hypertension; IRB: Institutional Review Board; NHLBI: National Heart, Lung, and Blood Institute; NIH: National Institute of Health; NSF: National Sleep Foundation; OSA: Obstructive Sleep Apnea; PI: Principal Investigator; S-TOFHLA: Short Test of Functional Health Literacy in Adults; RA: Research Assistant;

TASHE: Tailored Approach to Sleep Health Education

\section{Acknowledgements}

Not applicable.

\section{Funding}

This research is supported by the National Heart, Lung, and Blood Institute (NHLBI) (R25HL116378). Dr. Williams is supported by K23HL125939 from NHLBI; Dr. Ogedegbe is supported by K24 HL111315 from NHLBI. The study funder had no involvement in the study design, collection, management, analysis, and interpretation of data, or writing of the manuscript.

\section{Availability of data and materials}

The DSMP internal committee will review all requests for data before access to the data is granted and it may be made available (where appropriate) once the main findings of the project have been published.

\section{Authors' contributions}

GJL conceived of the protocol. GJL, GO, JPA, DR, AC, and NJW conceived and designed the manuscript. GJL, JPA, DR, and GO helped with financial support. NJW, RR, and GJL drafted the manuscript. GO, JPA, DR, AC revised the manuscript critically for important intellectual content. All authors read and approved the final manuscript.

Competing interests

The authors declare that they have no competing interests.

Consent for publication

Not applicable. 


\section{Ethics approval and consent to participate}

This study has received human ethics approval by the NYU School of Medicine Institutional Review Board (IRB \# i13-01011). The protocol is in full compliance with the standards set forth by the NYU School of Medicine IRB. This includes plans for communicating important protocol modifications, obtaining consent, and confidentiality. No study procedures will be conducted without informed written consent from the individual.

\section{Author details}

'Department of Population Health, Division of Health and Behavior, Center for Healthful Behavior Change, NYU Medical Center, New York, NY 10016, USA. ${ }^{2}$ Ichan School of Medicine at Mount Sinai, Pulmonary, Critical Care and Sleep Medicine, New York, NY 10029, USA. ${ }^{3}$ Department of Health and Behavior Studies, Teachers College, Columbia University, New York, NY 10027, USA. ${ }^{4}$ Harlem Health Promotion Center, Columbia University, New York, NY 10032, USA

Received: 19 April 2016 Accepted: 14 November 2016

Published online: 08 December 2016

\section{References}

1. Redline $\mathrm{S}$, et al. Racial differences in sleep-disordered breathing in AfricanAmericans and Caucasians. Am J Respir Crit Care Med. 1997;155(1):186-92.

2. Wallace DM, et al. The association of age, insomnia, and self-efficacy with continuous positive airway pressure adherence in black, white, and Hispanic U.S. Veterans. J Clin Sleep Med. 2013;9(9):885-95.

3. Platt $A B$, et al. Neighborhood of residence is associated with daily adherence to CPAP therapy. Sleep. 2009;32(6):799-806.

4. Billings $M E$, et al. Race and residential socioeconomics as predictors of CPAP adherence. Sleep. 2011;34(12):1653-8.

5. Dudley K, Patel S. Disparities and genetic risk factors in obstructive sleep apnea. Sleep Med. 2016;18:96-102.

6. Riley RW, et al. Obstructive sleep apnea. Trends in therapy. West J Med. 1995; 162(2):143-8.

7. Zhao YY, Redline S. Impact of continuous positive airway pressure on cardiovascular risk factors in high-risk patients. Curr Atheroscler Rep. 2015; 17(11):62.

8. CfDCa P. National Healthy Sleep Awareness Project. 2014. Available from: http://www.cdc.gov/sleep/projects partners.htm. Accessed 1 Dec 2016.

9. Ford ES, et al. Trends in outpatient visits for insomnia, sleep apnea, and prescriptions for sleep medications among US adults: findings from the National Ambulatory Medical Care Survey 1999-2010. Sleep. 2014;37(8): 1283-93.

10. Peppard $\mathrm{P}$, et al. Prospective study of the association between sleepdisordered breathing and hypertension. N Engl J Med. 2000;342:1378-84.

11. Aloia MS, et al. Neuropsychological sequelae of obstructive sleep apneahypopnea syndrome: a critical review. J Int Neuropsychol Soc. 2004;10(5):772-85.

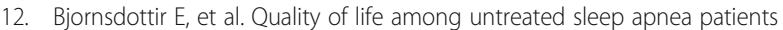
compared with the general population and changes after treatment with positive airway pressure. J Sleep Res. 2015;24(3):328-38.

13. Parker R, Ratzan SC. Health literacy: a second decade of distinction for Americans. J Health Commun. 2010;15:20-33.

14. Berkman ND, et al. Literacy and health outcomes. Evid Rep Technol Assess (Summ). 2004:87:1-8.

15. Berkman ND, et al. Low health literacy and health outcomes: an updated systematic review. Ann Intern Med. 2011;155(2):97-107.

16. DeWalt DA, et al. Literacy and health outcomes - a systematic review of the literature. J Gen Intern Med. 2004;19(12):1228-39.

17. Li JJ, et al. The relationship between functional health literacy and obstructive sleep apnea and its related risk factors and comorbidities in a population cohort of men. Sleep. 2014;37(3):571-8.

18. Sweet LH, Jerskey BA, Aloia MS. Default network response to a working memory challenge after withdrawal of continuous positive airway pressure treatment for obstructive sleep apnea. Brain Imaging Behav. 2010;4(2):155-63.

19. Sawyer AM, et al. A systematic review of CPAP adherence across age groups: Clinical and empiric insights for developing CPAP adherence interventions. Sleep Med Rev. 2011:15(6):343-56.

20. Wheaton $A G$, et al. Sleep disordered breathing and depression among U.S. adults: National Health and Nutrition Examination Survey, 2005-2008. Sleep. 2012;35:461-7.
21. Ghiassi R, et al. Developing a pictorial Epworth Sleepiness Scale. Thorax. 2011;66(2):97-100.

22. Chesson AL, et al. Presentation and reading level of sleep brochures: are they appropriate for sleep disorders patients? Sleep. 1998;21(4):406-12.

23. Baker DW, et al. Development of a brief test to measure functional health literacy. Patient Educ Couns. 1999:38(1):33-42.

24. Cheatham CT, Barksdale DJ, Rodgers SG. Barriers to health care and health-seeking behaviors faced by Black men. J Am Acad Nurse Pract. 2008;20(11):555-62

25. Green PM, Kelly BA. Colorectal cancer knowledge, perceptions, and behaviors in African Americans. Cancer Nurs. 2004;27(3):206-15.

26. Lewis LM, et al. Medication adherence beliefs of community-dwelling hypertensive African Americans. J Cardiovasc Nurs. 2010;25(3):199-206.

27. Ogedegbe $\mathrm{G}$, et al. Barriers and facilitators of medication adherence in hypertensive African Americans: a qualitative study. Ethn Dis. 2004;14(1):3-12.

28. Taylor KL, et al. Educating African American men about the prostate cancer screening dilemma: a randomized intervention. Cancer Epidemiol Biomarkers Prev. 2006:15(11):2179-88.

29. Ramos AR, et al. Associations between sleep disturbances and diabetes mellitus among blacks with metabolic syndrome: results from the Metabolic Syndrome Outcome Study (MetSO). Ann Med. 2015;47(3):233-7.

30. Demede $M$, et al. Resistant hypertension and obstructive sleep apnea in the primary-care setting. Int J Hypertens. 2011;2011:340929.

31. Shaw $R$, et al. Beliefs and attitudes toward obstructive sleep apnea evaluation and treatment among blacks. J Natl Med Assoc. 2012;104(11-12):510-9.

32. Williams $\mathrm{N}$, et al. I put the machine on and I sleep like a baby... a culturally and linguistically tailored telephone-behavioral intervention to increase adherence to sleep apnea recommendations among blacks with metabolic syndrome. Sleep. 2014;A59.

33. Minkler $M$, et al. Community-based participatory research: implications for public health funding. Am J Public Health. 2003:93(8):1210-3.

34. Israel BA, et al. Community-based participatory research: a capacity-building approach for policy advocacy aimed at eliminating health disparities. Am J Public Health. 2010;100(11):2094-102.

35. Kellogg W. WK Kellogg Foundation Evaluation Handbook. Battle Creek: WK Kellogg Foundation; 1998.

36. Lindenmeyer $\mathrm{A}$, et al. Assessment of the benefits of user involvement in health research from the Warwick Diabetes Care Research User Group: a qualitative case study. Health Expect. 2007;10(3):268-77.

37. Sung NS, et al. Central challenges facing the national clinical research enterprise. JAMA. 2003;289(10):1278-87.

38. Westfall JM, Mold J, Fagnan L. Practice-based research - "Blue Highways" on the NIH roadmap. JAMA. 2007;297(4):403-6.

39. Carney $C E$, et al. Beliefs about sleep in disorders characterized by sleep and mood disturbance. J Psychosom Res. 2007;62(2):179-88.

40. Murphy PW, et al. Comparing the effectiveness of video and written material for improving knowledge among sleep disorders clinic patients with limited literacy skills. South Med J. 2000:93(3):297-304.

41. Kreuter MW, Strecher VJ, Glassman B. One size does not fit all: the case for tailoring print materials. Ann Behav Med. 1999:21(4):276-83.

42. Levendowski DJ, et al. Prevalence of probable obstructive sleep apnea risk and severity in a population of dental patients. Sleep Breath. 2008;12(4):303-9.

43. Johns MW. A new method for measuring daytime sleepiness - The Epworth Sleepiness Scale. Sleep. 1991;14(6):540-5.

44. Smith $\mathrm{S}$, et al. Two new tools for assessing patients' knowledge and beliefs about obstructive sleep apnea and continuous positive airway pressure therapy. Sleep Med. 2004;5(4):359-67.

45. Rogers ES, et al. Assessing readiness for change among persons with severe mental illness. Community Ment Health. 2001;37:97-112.

46. Mastin DF, Bryson J, Corwyn R. Assessment of sleep hygiene using the sleep hygiene index. J Behav Med. 2006;29(3):223-7.

47. Belton $\mathrm{L}$, et al. Developing a scale to assess sleep apnea health literacy. Sleep. 2016; A0295.

48. Eastin M, LaRose R. Internet self-efficacy and the psychology of the digital divide. J Comput-Mediated Comm. 2000;6(1).

49. Sasson C, et al. A qualitative study to identify barriers to local implementation of prehospital termination of resuscitation protocols. Circ Cardiovasc Qual Outcomes. 2009;2(4):361-8.

50. Damush TM, et al. Barriers and facilitators to exercise among stroke survivors. Rehabil Nurs. 2007;32(6):253-60.

51. Cohen J. Statistical power analysis for the behavioral sciences. Orlando: Academic Press; 1977 
52. Fleiss J. Statistical methods for rates and proportions. 2nd ed. New York: Wiley; 1981.

53. Ibrahim JG, Chen MH, Lipsitz SR. Monte Carlo EM for missing covariates in parametric regression models. Biometrics. 1999;55(2):591-6.

54. Kilbourne AM, et al. Advancing health disparities research within the health care system: a conceptual framework. Am J Public Health. 2006;96(12):2113-21.

55. Hackney JE, Weaver TE, Pack Al. Health literacy and steep disorders: a review. Sleep Med Rev. 2008;12(2):143-51.

56. Krishna S, Boren SA, Balas EA. Healthcare via cell phones: a systematic review. Telemed J E Health. 2009;15(3):231-40.

57. Noh JH, et al. Web-based comprehensive information system for selfmanagement of diabetes mellitus. Diabetes Technol Ther. 2010;12(5):333-7.

58. Goodall K, Ward P, Newman L. Use of information and communication technology to provide health information: what do older migrants know, and what do they need to know? Qual Prim Care. 2010;18(1):27-32.

59. Khan SA, et al. GetHealthyHarlem.org: developing a web platform for health promotion and wellness driven by and for the Harlem community. AMIA Annu Symp Proc. 2009;2009:317-21.

60. Cohall AT, et al. Computer use, internet access, and online health searching among Harlem adults. Am J Health Promot. 2011;25(5):325-33.

61. Cohall A, et al. HIV testing preferences among young men of color who have sex with men. Am J Public Health. 2010;100(10):1961-6.

\section{Submit your next manuscript to BioMed Central} and we will help you at every step:

- We accept pre-submission inquiries

- Our selector tool helps you to find the most relevant journal

- We provide round the clock customer support

- Convenient online submission

- Thorough peer review

- Inclusion in PubMed and all major indexing services

- Maximum visibility for your research

Submit your manuscript at www.biomedcentral.com/submit 